\title{
- REPORTE DE CASO
}

\section{Escherichia coli metalobetalactamasa en un hospital de alta complejidad en Paraguay Escherichia coli metallobetalactamase in a highly complex Hospital in Paraguay}

\author{
Autores: Myrian Rivas Kiese ${ }^{1}$, Helena Ortiz ${ }^{1}$, Patricia Almada ${ }^{1}$, Rocío Arguello ${ }^{1}$, \\ Nancy Melgarejo ${ }^{2}$, Celia Martínez ${ }^{3}$, Zunilda Garay ${ }^{3}$ \\ Artículo recibido: 27 junio 2016 Artículo aceptado: 25 julio 2016
}

\begin{abstract}
Resumen
Ante la aparición de Escherichia coli con New Delhi metalobetalactamasa en un hospital de alta complejidad en Paraguay, se resalta la importancia de vigilar y detectar mecanismos de resistencias así como establecer oportunamente medidas de prevención y control de la infección por microorganismos multirresistentes causantes de brotes y relacionados con un incremento en la morbilidad y mortalidad asociada a los cuidados de salud.
\end{abstract}

Palabras clave: Escherichia coli, carbapenémicos, farmacorresistencia microbiana.

\begin{abstract}
With the emergence of Escherichia coli with New Delhi metallobetalactamase in a hospital of high complexity in Paraguay, the importance of monitoring and detecting resistance mechanisms is highlighted and is consider timely to establish measures for prevention and control of multiresistent microorganisms that causes an outbreak of infections They are related with an increased of morbidity and mortality associated with health care.
\end{abstract}

Keywords: Escherichia coli, carbapenems, microbial drug resistance

\section{Introducción}

El hallazgo de enterobacterias resistentes a carbapenemes, como consecuencia de la adquisición de carbapenemasas, es un alarmante problema mundial asociado a elevadas tasas de mortalidad, altos niveles de resistencia a otros antimicrobianos y gran potencial de diseminación ${ }^{1,2}$. Desde 2008 se ha documentado a nivel mundial la circulación de microorganismos con mecanismo de resistencia antimicrobiana denominado "New Delhi metalobetalactamasa" (NDM), un tipo de carbapenemasas que ocasiona resistencia a todos los antibióticos betaláctamicos excepto aztreonam ${ }^{3}$.

${ }^{1}$ Departamento de Bacteriología. Hospital de Clínicas. Facultad de Ciencias Médicas, U.N.A.

${ }^{2}$ Laboratorio Central del Ministerio de Salud Pública

${ }^{3}$ Control de Infecciones Intrahospitalarias. Hospital de Clínicas. Facultad de Ciencias Médicas, U.N.A.

\section{Autor correspondiente:}

Nombres y apellidos: Dra. Myrian Rivas Kiese

Dirección: Rolón Viera 1340. Asunción, Paraguay

Teléfono: +(595) 981418151

Correo electrónico:myrianrivas23@yahoo.com.mx 
En 2010 se aisla en Australia la primera Escherichia coli productora de carbapenemasa ${ }^{4}$. Los primeros hallazgos en las Américas se registraron en 2010 en Estados Unidos de América y Canadá, en pacientes que tenían antecedentes de haber recibido atención médica reciente en países fuera de la región. Posteriormente, en 2011, este mecanismo de resistencia fue detectado en Guatemala. En 2012 se detectó en Colombia en Klebsiella pneumoniae, en Paraguay en Acinetobacter pittii y en Uruguay en Providencia rettgeri. En 2013 otros países habían reportado el hallazgo de la circulación de este mecanismo: Argentina en P. rettgeri, Brasil en $P$. rettgeri, Honduras en $A$. baumannii, México en P. rettgeri, Nicaragua en A. baumannii, K. pneumoniae, E. coli y Enterobacter cloacae; y, recientemente, Costa Rica en E. coli ${ }^{3}$.

La producción y diseminación de carbapenemasas se produce por medio de plásmidos por lo que el riesgo de diseminación entre especies es muy alto ${ }^{5}$. Esto ha permitido que una carbapenemasa de clase $\mathrm{B}$ gane la atención de los medios de salud desde que se la identificó por primera vez en Klebsiella pneumoniae y Escherichia coli en India y Pakistán en el $2008^{6}$.

\section{Caso clínico}

Paciente de sexo femenino, de 22 años de edad, portadora de aplasia medular probablemente secundaria a hepatitis con carga viral negativa, en tratamiento con ciclosporina, presentó tres internaciones previas en el Hospital de Clínicas (San Lorenzo, Paraguay) por infección de piel y partes blandas. El motivo de su internación actual es por un cuadro de fiebre, gingivorragia y proceso infeccioso en muslo derecho.

Se tomaron muestras clínicas, con aislamiento de Klebsiella pneumoniae en tratamiento con ceftriaxona. Se registra una intervención quirúrgica para desbridamiento de fascitis necrotizante en región perianal y tras 12 días de internación se toma muestra de hemocultivo en la que se aisla Escherichia coli, con el mismo patrón de resistencia que la cepa aislada de la muestra obtenida de la herida operatoria. Tanto la identificación de la cepa como el perfil de resistencia fue obtenido utilizando el equipo automatizado Vitek 2 Compact (bioMe'rieux Clinical Diagnostics, France). Ambos aislamientos resultaron resistentes a carbapenemes con una concentración inhibitoria mínima (CIM) $>16 \mu \mathrm{g} / \mathrm{mL}$ para imipenem y meropenem. Por tal motivo, se realiza el test de Hodge modificado (MHT) y se remite la cepa al Laboratorio Central del Ministerio de Salud Pública, donde se confirma la presencia de New Delhi Metalobetalactamasa (NDM1). A las 2 semanas de su internación ingresa a la unidad de cuidados intensivos por cuadro de insuficiencia respiratoria aguda y fallece al cuarto día de internación. El aislamiento de Escherichia coli MBL es el primer caso descrito en el Hospital de Clínicas y de ahí radica de vital importancia evitar la diseminación de este germen con su particular mecanismo de resistencia en la población general.

\section{Discusión}

Los carbapenemes son frecuentemente los antibióticos utilizados para tratamientos de infecciones por enterobacterias multirresistentes ${ }^{6}$, por lo que la circulación en el país de cepas NDM1 constituye un grave problema de salud pública ${ }^{7}$. Su emergencia en asociación con otros genes de resistencia demuestra la rápida evolución de la bacteria y su habilidad para adquirir y mantener diferentes genes de resistencia ${ }^{6}$. Esto preocupa ya que las opciones de tratamiento disponibles disminuyen, pues como en este caso, la cepa mostraba susceptibilidad a colistina solamente (CIM $\leq 0,5 \mu \mathrm{g} / \mathrm{mL})$ siendo resistente a todos los demás antimicrobianos testados.

Ante la detección en varios países de la región de las Américas de microorganismos con mecanismos de 
resistencia a la colistina a través de plásmidos $m c r$ - 1 , aislados tanto en animales como en humanos, la OPS/OMS urgen a los estados miembros a implementar y mantener la capacidad para detectar, prevenir y controlar la transmisión de microorganismos con resistencia transferible a colistina y a tomar medidas que conduzcan a prohibir el uso de estos antimicrobianos como profilaxis y promotores de crecimiento en animales destinados al consumo humano ${ }^{8}$.

La resistencia microbiana es un problema creciente con impacto múltiple en la atención sanitaria ya que prolonga la duración de la enfermedad infecciosa, aumenta las interacciones farmacológicas, los costos asistenciales, la carga económica sobre las familias y la sociedad y el riesgo de muerte?

Entre las medidas recomendadas por el CDC en la guía para el control de las enterobacterias resistentes a carbapenémicos se encuentra realizar vigilancia de enterobacterias productoras de carbapenemasas a los contactos de un caso índice conocido e implementar precauciones de contacto precoces ante la sospecha. Además, en hospitales sin casos descritos, que reciben pacientes transferidos desde centros con casos de pacientes colonizados o infectados con enterobacterias productoras de carbapenemasas, se recomienda efectuar un tamizaje en el momento de la admisión e implementar precauciones de contacto en espera del resultado de los cultivos de vigilancia ${ }^{10}$.

Hasta ahora, Acinetobacter spp no baumannii que expresa NDM no ha sido descrito en el continente americano. Los aislamientos clínicos de A. pittii productores de NDM1 de Paraguay fueron los primeros. Su origen no es claro ya que no hay antecedentes de viajes en estos pacientes ${ }^{11}$. Datos publicados recientemente sugieren que $A$. pittii podría actuar como un reservorio potencial de NDM para Enterobacterias basados en la facilidad de la transferencia de plásmido a $E_{\text {.coli }}{ }^{12}$.

Esto es de suma importancia ya que el aislamiento de E. coli NDM en cuestión es realizada en el mismo hospital donde se había registrado $A$. pittii por lo que se insiste en la vigilancia conjunta a través del Laboratorio de Microbiología y el Departamento de Control de Infecciones Nosocomiales para una rápida respuesta y aislamiento de pacientes para evitar la difusión de las mismas.

Las cepas productoras de carbapenemasas de tipo KPC se han diseminado por todo el mundo y Paraguay no es una excepción. Debido a los factores de riesgo vinculados a los cuidados de la salud en áreas cerradas y con procedimientos invasivos así como al uso de antibioticoterapia previa, constituye todo un desafío diagnóstico y terapéutico dado las escasas opciones disponibles, lo que obliga a optimizar el uso de los antimicrobianos así como a realizar combinaciones sinérgicas de los mismos que sólo en cierta medida han demostrado eficacia.

Se debe continuar la vigilancia activa de estas enzimas tanto a nivel comunitario como hospitalario, ya que conocer la realidad local permitirá tomar medidas adecuadas y estar alertas ante la llegada de nuevas carbapenemasas en diferentes enterobacterias, los que constituye un desafío clínico, diagnóstico y terapéutico. 


\section{Referencias bibliográficas}

1. Gupta N, Limbago BM, Patel JB, Kallen AJ. Carbapenem-resistant Enterobacteriaceae: epidemiology and prevention. Clin Infect Dis. 2011 Jul 1;53(1):60-7.

2. Gutiérrez C, Labarca J, Román JC, Sanhueza F, Moraga M, Wozniak A, García P. Vigilancia de enterobacterias productoras de carbapenemasas en cultivos rectales en un hospital universitario de Santiago, Chile. Rev Chilena Infectol. 2013 Feb; 30(1):103-6.

3. Organización Panamericana de la Salud, Organización Mundial de la Salud. Actualización epidemiológica. Carbapenemasas tipo New Nelhi metalobetalactamasas (NDM) /Internet/: OPS-OMS; 2014. /citado 3 Oct. 2015/. Disponible en: http://www.paho.org/hq/index.php?option=com_docman\&task=doc view\&gid $=24472$

4. Poirel L, Lagrutta E, Taylor P, Pham J, Nordmann P. Emergence of metallo- $\beta$-lactamase NDM-1-producing multidrug-resistant Escherichia coli in Australia. Antimicrob Agents Chemother. 2010; 54(11):4914-6.

5. Organización Panamericana de la Salud, Organización Mundial de la Salud. Alerta epidemiológica: Primer hallazgo de carbapenemasas de tipo New Delhi metalobetalactamasas (NDM) en Latinoamérica. /Internet/. 2011. /citado 3 Oct. 2015/. Disponible en: http://antimicrobianos.com.ar/ATB/wpcontent/uploads/2013/01/22_noviembre_2011_carbapenemasas_americas1.pdf

6. Quiles MG, Rocchetti TT, Fehlberg LC, Kusano EJ, Chebabo A, Pereira RM,et al. Unusual association of NDM1 with KPC-2 and armA among Brazilian Enterobacteriaceae isolates. Braz J Med Biol Res. 2015; 48(2):1747.

7. Liu C, Qin S, Xu H, Xu L, Zhao D, Liu X, et al. New Delhi Metallo- $\beta$-Lactamase 1(NDM-1), the Dominant Carbapenemase Detected in Carbapenem-Resistant Enterobacter cloacae from Henan Province, China. PLoS One. 2015 Aug 11; 10(8):e0135044.

8. Organización Panamericana de la Salud, Organización Mundial de la Salud. Alerta Epidemiológica: Enterobacterias con resistencia transferible a colistina, implicaciones para la salud pública en las Américas.Washington, D.C. : OPS/OMS. 2016.

9. Lastra Vde L, Rivas LM, Silva F, Ulloa MT, Pinto ME, Vidal M. Detección de serinocarbapenemasas de clase A y otros mecanismos de resistencia enzimática a $\beta$-lactámicos en cepas de enterobacterias con susceptibilidad disminuida a carbapenémicos, aisladas de pacientes de un hospital universitario de Santiago, Chile. Rev. Chil. Infectol. 2014; 31(6):682-8.

10. National Center for Emergind and Zoonotic Infectious Diseases. Facility guidance for Control of Carbapenem-resistant Enterobacteriaceae (CRE). /Internet/. /citado 3 Nov. 2015/: National Center for Emergind and Zoonotic Infectious Diseases; 2015. Disponible en: http://www.cdc.gov/hai/pdfs/cre/CREguidance-508.pdf

11. Pasteran F, Mora MM, Albornoz E, Faccone D, Franco R, Ortellado J, et al. Emergence of genetically unrelated NDM-1-producing Acinetobacter pittii strains in Paraguay. J Antimicrob Chemother. 2014; 69(9):2575-8.

12. Bogaerts $P$, Huang TD, Rezende de Castro R, Bouchahrouf W, Glupczynski Y. Could acinetobacter pittii act as an NDM-1 reservoir for Enterobacteriaceae?. J Antimicrob Chemother. 2013; 68(10):2414-5. 